\title{
One-pot synthesis and characterization of InP/ZnSe semiconductor nanocrystals
}

\author{
Paul Mushonga, Martin O. Onani, Abram M. Madiehe and Mervin Meyer
}

\begin{abstract}
We report on the one-pot synthesis of InP/ZnSe quantum dots (QDs) using a precursor-based colloidal synthesis in a noncoordinating solvent environment. The structural and optical studies were carried out on the as-prepared InPQDs. The quantum yield of the nanocrystals was recorded as $6 \%$ and a 1.4 times reduction in the ratio of traprelated emission to band edge emission was observed on $\mathrm{ZnSe}$ passivation of the InPQDs.
\end{abstract}

\section{Introduction}

The colloidal group III-V semiconductor nanocrystals (NCs) have attracted enormous interest in the past two decades [1]. The principal attraction to this class of nanocrystals lies in the robustness of their covalent bond compared to the ionic bond in group II-VI semiconductor systems $[2,3]$. This property carries along an enhanced optical stability and reduced toxicity as a result of non-erosion of constituent ions when they are used in biological milieu [4]. Within the group III$\mathrm{V}$ semiconductor materials, the InP system appears popular due to its relatively narrow band gap (1.35 eV) and large excitonic Bohr radius (11.3 nm) [5]. These parameters allow for the tuning of the photoluminescence emission wavelengths from the blue to the near infrared regions [6]. Research in InP-based nanocrystals is also premised on the need to find less toxic non-cadmium based quantum dots for applications in biomedicine [7].

However, despite the fact that the covalent nature of the group III-V is acknowledged, it is unfavorable for their synthesis. The precursors of group III-V nanocrystals possess strong covalent bonding, and therefore demand high synthetic temperatures and long reaction times. In 2010, Kim et al. [8] reported the fabrication of trioctylphosphine oxide (TOPO)-capped InP/ZnSe using chloroindium oxalate as an indium precursor in a reaction carried out over several days with a quantum yield of 6.8. In this paper, we report a shorter route to the synthesis of palmitic acidcapped InP/ZnSe nanocrystals using a one-pot hot-injection [9] method. Further, weakly coordinating solvent, 1-octadecene (ODE), was used in our work because 
strongly coordinating solvents lead to slow and continuous nucleation, thus making it difficult to attain discrete nucleation and growth steps which are essential in obtaining monodisperse quantum dots [5].

\section{Experimental}

The solvents used (acetone and $n$-hexane) were CHROMASOLV HPLC grade and were used as received from Sigma Aldrich. Indium acetate $\left(\operatorname{In}(\mathrm{OAc})_{3}\right)$, palmitic acid, zinc undecylenate, selenium, trioctylphosphine (TOP), 1-octadecene (ODE) and tris-(trimethylsilyl)phosphine $\left(\mathrm{P}(\mathrm{TMS})_{3}\right)$ were purchased from Sigma Aldrich.

Synthesis of InP/ZnSe NCs: The NCs were synthesized in a non-coordinating solvent, 1-octadecene according to the procedure by Ryuet al. with some modifications [10]. In a typical synthesis, indium acetate $(0.15 \mathrm{mmol})$ was mixed with palmitic acid $(0.45 \mathrm{mmol})$ and ODE $(4 \mathrm{~mL})$ in a three-necked flask in the glove box. The flask was sealed and transferred to a Schlenk line and heated to $120{ }^{\circ} \mathrm{C}$ and kept under vacuum for $1.5 \mathrm{~h}$. The system was then purged with argon and then heated to $300{ }^{\circ} \mathrm{C}$. On reaching $300{ }^{\circ} \mathrm{C}$, a freshly prepared injection solution of $\mathrm{P}(\mathrm{TMS})_{3}$ in ODE (0.075 $\mathrm{mmol}$ in $1 \mathrm{~mL} \mathrm{ODE} \mathrm{prepared} \mathrm{in} \mathrm{the} \mathrm{glove} \mathrm{box)}$ was injected rapidly under argon flow. For the growth of the InP core, the reaction mixture was kept at $270{ }^{\circ} \mathrm{C}$ temperature was lowered to $150{ }^{\circ} \mathrm{C}$. For the shelling process, the zinc injection solution $(0.1 \mathrm{M})$ was prepared by dissolving zinc undecylenate in a mixture of ODE $(9.5 \mathrm{~mL})$ and TOP $(0.5 \mathrm{~mL})$ at $140{ }^{\circ} \mathrm{C}$. Similarly the selenium injection solution (0.1 M) was prepared by dissolving selenium in a mixture of ODE $(9.5 \mathrm{~mL})$ and TOP $(0.5 \mathrm{~mL})$ at $1401 \mathrm{C}$. The zinc precursor solution $(0.375 \mathrm{mmol}, 0.375 \mathrm{~mL})$ was added and the temperature was raised to $2301 \mathrm{C}$ and maintained at that temperature for $4 \mathrm{~h}$. A selenium precursor solution $(0.15 \mathrm{mmol}, 1.5 \mathrm{~mL})$ was injected into the flask and kept at this temperature for $1 \mathrm{~h}$. Aliquot portions were taken during core and shell growth for characterization. The InP/ZnSe nanocrystals were purified from the reaction mixture by precipitation with acetone and redissolving them in $n$-hexane.

Characterization Optical measurements: Photoluminescence (PL) spectra were recorded on a HORIBA Nanolog FL3-22-TRIAX. The photoluminescence quantum yield (QY) for the InP/ZnSe NCs was determined using Rhodamine 6G as a standard according to a published procedure [11]. The optical densities of all the solutions were adjusted to values below 0.05 at the excitation wavelength of 400 $\mathrm{nm}$ to avoid re-absorption effects. The absorbances for the standard in ethanol and the InP/ZnSe in hexane at this excitation wavelength and the photoluminescence spectra of the same solutions were measured respectively. The integrated fluorescence intensity from the corrected spectrum was calculated. 
Transmission electron microscopy (TEM): TEM studies were performed on a TECNAI F30ST TEM. Samples for TEM studies were prepared by placing a hexane solution of the nanocrystals on ultrathin carbon-film-coatedcopper grids.

\section{Results and discussion}

Optical properties of the InP/ZnSe QDs: Highly fluorescent and monodisperse $\mathrm{InP} / \mathrm{ZnSe}$ nanocrystals were synthesized by reacting indium acetate with tris(trimethylsilyl phosphine) $\left(\mathrm{P}(\mathrm{TMS})_{3}\right)$ using palmitic acid (PA) as the surfactant and 1-octadecene as the noncoordinating solvent. Palmitic acid, an intermediate chain length fatty acid, facilitates controlled nucleation and growth rates needed for the synthesis of monodisperse InP semiconductor nanocrystals in non-coordinating solvents [12]. We maintained the 1:3 ratio of indium precursor to surfactant as it yields high-quality nanocrystals [13]. Fig. 1 shows normalized photoluminescence (PL) spectra of three aliquots taken during the synthesis of the NCs. The spectra show that there is no significant change in the emission peak maximum for aliquots taken $30 \mathrm{~min}$ and $120 \mathrm{~min}$ into the core growth. Bawendi's group reported that in the synthesis of InP, molecular phosphorus precursors are completely used up at the InP nucleation step culminating in subsequent growth of nanocrystals exclusively being attributed to ripening from non-molecular InP species in the solution [14].

The as-prepared InP/ZnSe NCs showed a maximum emission wavelength at $574 \mathrm{~nm}$. An aliquot taken 120 min into core growth gave an emission peak of $579 \mathrm{~nm}$. The slight blue-shift observed for these type I core/shell systems is contrary to expectations and is attributed to the fact that photoluminescence in $\mathrm{ZnSe}$ shelled-InP is less contributed by defect emission in comparison to the bare InP NCs [7]. The photoluminescence quantum yield (QY) was measured as $6 \%$.

Trap state-related emissions are also exhibited in the PL spectra. The surface states are a result of unsatisfied (dangling) bonds that are found at the reconstructed surface. In this work, we chose $\mathrm{ZnSe}$ with a lower lattice mismatch with InP as a inorganic shell instead of $\mathrm{ZnS}$ which has a relatively large lattice mismatch in the zinc blende phase of about $7.6 \%$ [15]. Fig. 2 shows each PL spectrum deconvoluted into two Gaussian peaks. The deep traprelated emissions for the InP/ZnSe and bare InP NCs are around $627 \mathrm{~nm}$ and $640 \mathrm{~nm}$ respectively. The blue-shift observed is an evidence of successful coating of the core and that the shell reduces the surface states. The ZnSe shell also greatly reduced the ratio of traprelated emission to band edge emission by a factor of 1.4 [16].

Structural properties of the InP/ZnSe QDs: Figs. 3 and 4 show the HRTEM micrographs and EDX results of the nanocrystals respectively. The InP/ZnSe nanocrystals had an average diameter of $1.95 \mathrm{~nm}$ and the lattice fringes in the HRTEM image confirm the good crystallinity of the nanocrystals with lattice-fringe distance of $0.29 \mathrm{~nm}$ (nanocrystals in white dotted circles) which is in good agreement with the plane spacing of $0.29343 \mathrm{~nm}$ for /20oS planes of the zinc blende structure. These lattice fringes are continuous throughout the QD indicating the epitaxial nature of ZnSe shell growth [17]. The selected area electron diffraction (SAED) pattern (Inset, Fig. 3) confirmed the zinc blende structure 
of the NCs with three rings indexed to /111S, /220S and /311S lattice planes with corresponding calculated $d$-spacing values of $0.3404,0.2069$ and $0.17716 \mathrm{~nm}$ in good agreement with the theoretical ones. The EDXS results for the InP/ZnSe NC sample showed an In:P:Zn:Se ratio of 1:0.96:1.47:0.17 showing an indium-rich core with a zinc-rich particle surface. Thus the InP core has an indium-terminated surface while the shell has zinc-terminated surface. The $\mathrm{Zn} / \mathrm{In}$ ratio of 1.47 confirms that the InP cores are covered by a ZnSe shell. Even higher ratios (2.34) have been reported for ZnS-capped InP nanocrystals [18].

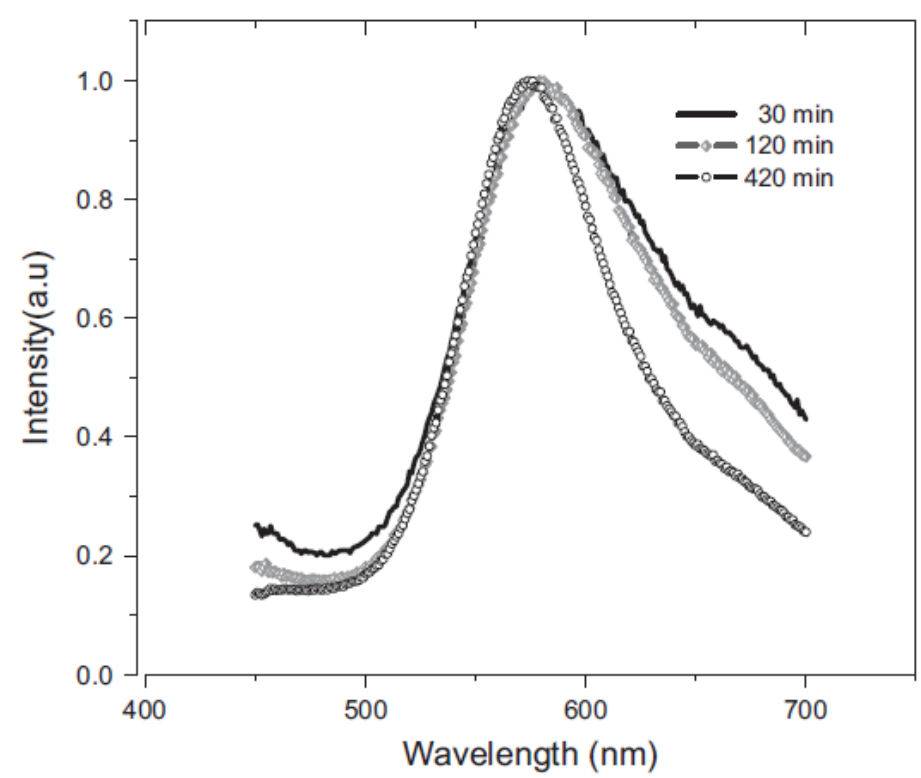

Fig. 1. Normalized photoluminescence spectra of three aliquots taken during the synthesis of the NCs.

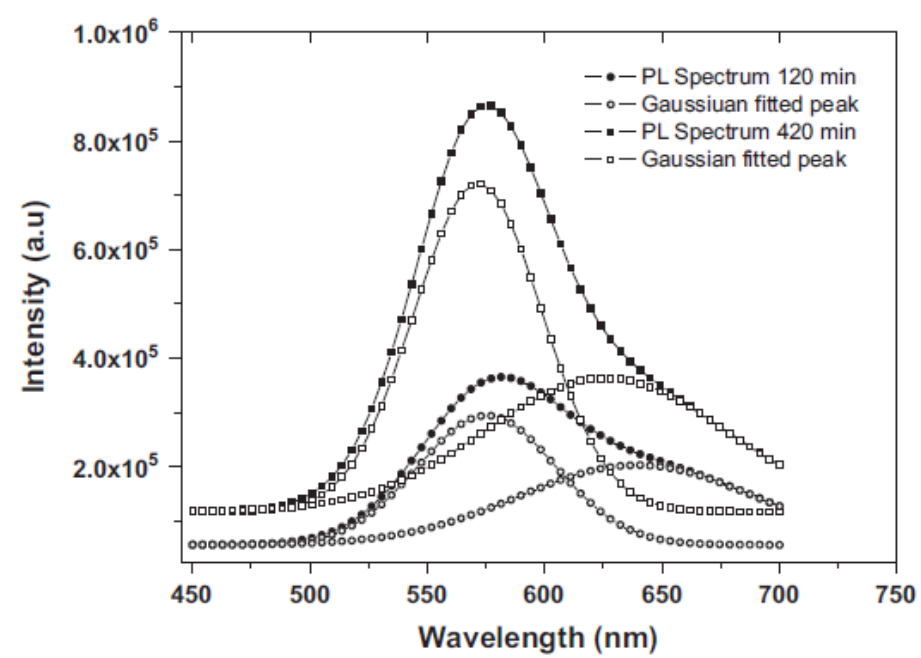

Fig. 2. Photoluminescence spectra deconvoluted into Gaussian peaks. 


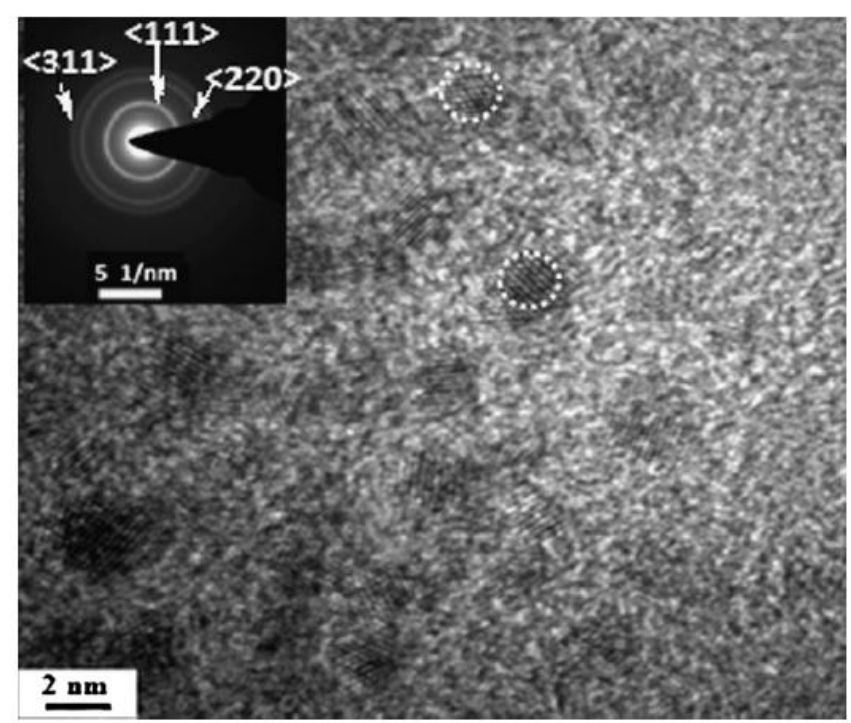

Fig. 3. HRTEM micrograph of the InP/ZnSe NCs (Insert is an SAED pattern).

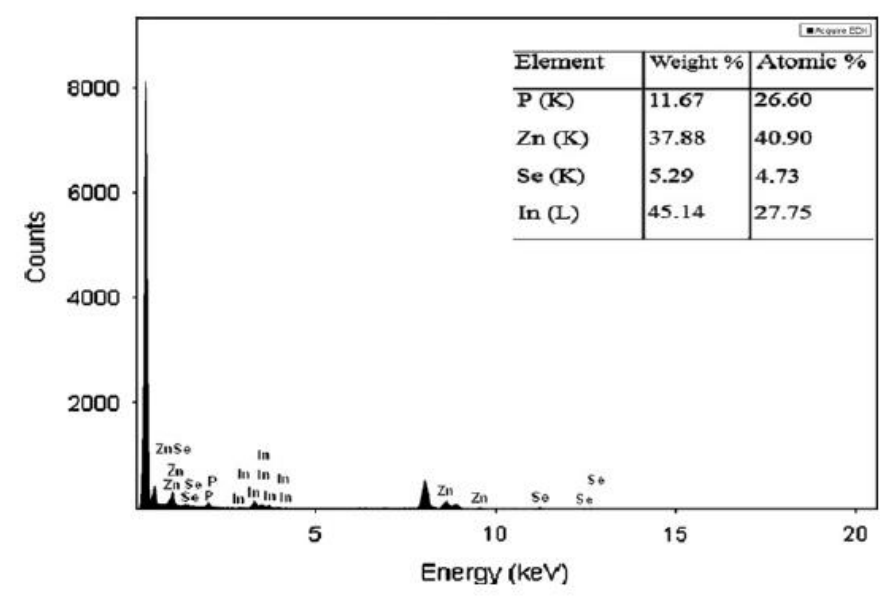

Fig. 4. EDX results of the InP/ZnSe NCs.

\section{Conclusion}

In conclusion, we have successfully synthesized InP/ZnSe QDs emitting at $574 \mathrm{~nm}$ with a QY of $6 \%$. We have also demonstrated that indeed ZnSe shell passivates the InP core QDs with a blue-shift in the emission profiles and a 1.4 times reduction in the ratio of trap-related emission to band edge emission. The nanocrystals will undergo ligand exchange reactions as a functionalization step before they are applied in biological imaging studies.

\section{Acknowledgments}

The authors acknowledge the NIC/DST and UWC Senate Research for financial support. 


\section{References}

[1] Pham TT, Tran TKC, Nguyen QL. Adv Nat Sci Nanosci Nanotechnol 2011;2(025001):1-4.

[2] Narayanaswamy A, Feiner LF, Van der Zaag PJ. J Phys Chem 2008;C 112(17):6775-80.

[3] Bharali DJ, Lucey DW, Jayakumar H, Pudavar HE, Prasad PN. J Am Chem Soc 2005;127(32):11364-71.

[4] Yong K-T, Ding H, Roy I, Law W-C, Bergey EJ, Maitra A, et al. ACS Nano 2009;3:502-10.

[5] Langof L, Fradkin L, Ehrenfreund E, Lifshitz E, Micic OI, Nozik AJ. Chem Phys 2004;297(1-3):93-8.

[6] Xu S, Ziegler J, Nann T. J Mater Chem 2008;18:2653-6.

[7] Paul Mushonga P, Onani MO, Madiehe AM, Meyer M. J Nanomater 2012;2012(869284):1-11.

[8] Kim MR, Chung JH, Lee M, Lee S, Du-Jang D-J. J Colloid Interface Sci 2010;350(1):5-9.

[9] Murray CB, Norris DJ, Bawendi MG. J Am Chem Soc 1993;115(19):870615. [10] Ryu E, Kim S, Jang E, Jun S, Jang H, Kim B, et al. Chem Mater 2009;21(4):573-5.

[11] Byun H-J, Song W-S, Yang H. Nanotechnology 2011;23(235605):1622 2011;23(235605):1-6.

[12] Battaglia D, Peng X. Nano Lett 2002;2(9):1027-30.

[13] Xu S, Kumar S, Nann T. J Am Chem Soc 2006;128(4):1054-5.

[14] Allen PM, Walker BJ, Bawendi MG. Angew Chem Int Ed 2010;49(4):760-2. [15] Huang K, Demadrille R, Silly MG, Sirotti F, Reiss P, Renault O. ACS Nano 2010;4(8):4799-805.

[16] Protiere M, Reiss P. Chem Commun 2007:2417-9.

[17] Gao S, Zhang C, Liu Y, Su H, Huang T, Dellas N, et al. Opt Exp 2011;19(6):5528-35.

[18] Byun H-J, Lee JC, Yang H. J Colloid Interface Sci 2011;355(1):35-41. 